\title{
Electron Charge Density Distribution from X-Ray Diffraction Study of the 4-Methoxybenzenecarbothioamide Compound
}

\author{
Mokhtaria Drissi, Abdelkader Chouaih, Youcef Megrouss, and Fodil Hamzaoui \\ Laboratoire de Technologie et Propriétés du Solide (LTPS), Faculté des Sciences et de la Technologie, \\ Université Abdelhamid Ibn Badis de Mostaganem, 27000 Mostaganem, Algeria
}

Correspondence should be addressed to Abdelkader Chouaih; achouaih@gmail.com

Received 31 March 2013; Accepted 1 July 2013

Academic Editors: N. Ishii and E. Suresh

Copyright (C) 2013 Mokhtaria Drissi et al. This is an open access article distributed under the Creative Commons Attribution License, which permits unrestricted use, distribution, and reproduction in any medium, provided the original work is properly cited.

\begin{abstract}
The molecular electron charge density distribution of the title compound is described accurately using the multipolar model of Hansen and Coppens. The net atomic charge and the in-crystal molecular dipole moment have been determined in order to understand the nature of inter- and intramolecular charge transfer. The study reveals the nature of intermolecular interactions including charge transfer and hydrogen bonds in the title compound. In this crystal, the molecules form dimers via $\mathrm{N}-\mathrm{H} \cdots \mathrm{S}$ intermolecular hydrogen bonds. The dimers are further linked by $\mathrm{C}-\mathrm{H} \cdots \mathrm{O}$ hydrogen bonds into chains along the $c$ crystallographic axis. This study has also allowed us to determine the electrostatic potential and therefore locate the electropositive part and the electronegative part in molecular scale of the title compound.
\end{abstract}

\section{Introduction}

The chemistry of thioamides and their derivatives continues to be of particular interest due to their interesting structural features and also due to their biological importance. Heterocyclic compounds of these types exhibit a wide spectrum of biological activity and have extensive practical applications [1-3]. In addition, thioamides are important ligands in the field of coordination chemistry [4]. Due to these positive traits, thioamides have been under study for a long time, but much of their basic chemistry remains unexplored.

The heterocyclic 4-methoxybenzenecarbothioamide compound, of chemical formula $\mathrm{C}_{8} \mathrm{H}_{9} \mathrm{NOS}$, crystallizes in the $\mathrm{P} 22_{1} 2_{1}$ space group, with eight molecules in the unit cell. The general features of the structure have been described previously [5]. It contains two independent molecules with the methoxy groups oriented in opposite conformations. The mean planes of the carbothioamide groups are tilted by $7.88(15)$ and $11.16^{\circ}(9)$ from the mean planes of the benzene rings.

In this work, the crystallographic data were taken from another article [5] and used for the current study. The structure has been redetermined using the same X-ray diffraction data.
So this work focuses on the thermal analysis and electronic charge density study of the 4-methoxybenzenecarbothioamide molecule. The scope of this study is to model aspherical features of the atomic electron density arising from chemical bonding at low temperature of the molecule by transferring the electron density multipole parameters obtained from accurate X-ray diffraction experiments and calculate the molecular dipole moment. In order to understand the nature of inter- and intramolecular charge transfer, the electrostatic potential distribution of the molecule in crystal has been analyzed.

\section{Materials and Methods}

2.1. High-Order Spherical Atom Refinement. A high-order spherical atom refinement $(\mathrm{HO})$ on the non- $\mathrm{H}$ atoms is performed to get least-biased positional and thermal parameters. Hence, due to the properties of the Fourier transform, this $\mathrm{HO}$ procedure refines the positional and anisotropic thermal motion parameters only on core electrons. Because valence electron scattering factors diffuse only at low resolution, $\mathrm{HO}$ refinement gives precise positions and displacements of the core electrons only. 
In order to extract the aspherical features of the electron density, the following strategy was designed. First, a fullanisotropic spherical atom refinement using starting parameters from a Shelxl [6] refinement is performed. Then, the multipole refinement which has been developed by Hansen and Coppens [7] is selected to perform the electron density analysis using the MoPro package software [8].

In parallel, the net atomic charge on the different atoms of the title compound and the molecular dipole moment were also estimated by theoretical calculations. Theoretical calculations were performed with the Gaussian package [9] at the HF/6-31G* level of theory and semiempirical methods.

A higher-order refinement of X-ray data becomes essential to obtain accurate core positions and the associated thermal parameters of the different atoms of the molecule.

The X-ray scattering factors for S, C, N, and $\mathrm{O}$ atoms come from the international tables for X-ray crystallography [10] while for $\mathrm{H}$ atom the bonded $\mathrm{H}$-atom scattering curve of Stewart et al. (1965) was used [11]. The $\mathrm{H}$ atoms were found by difference Fourier synthesis and their coordinates were adjusted by extending along the $\mathrm{C}-\mathrm{H}$ and $\mathrm{N}-\mathrm{H}$ bond directions to bond lengths of $1.105 \AA$ and $1.020 \AA$, respectively. Any position error of the hydrogen atom will bring about errors strongly correlated with the dipolar population of hydrogen. Refinement details are displayed in Table 1.

2.2. Charge Density Multipole Model. The Hansen-Coppens multipole formalism, as implemented in the Mopro leastsquares program for multipole refinement, was used for both observed and theoretical structure factor fitting. It describes the crystal electron density as a superposition of aspherical pseudo-atoms each modeled on a multipole expansion:

$$
\begin{aligned}
\rho_{\text {atom }}(\vec{r})= & \rho_{c}(\vec{r})+P_{V} K^{\prime 3} \rho_{v}\left(k^{\prime} \vec{r}\right) \\
& +\sum_{l}^{l \max } \sum_{m=-1}^{+1} K^{\prime \prime} R_{l}\left(K^{\prime \prime} \vec{r}\right) P_{l m} Y_{l m}\left(\frac{\vec{r}}{r}\right) .
\end{aligned}
$$

Here $\rho_{c}$ and $\rho_{v}$ are spherically averaged Hartree-Fock core and valence densities, with $\rho_{v}$ being normalized to one electron. The Slater-type radial functions $R_{l}(\vec{r})=N_{l} \cdot r_{l}^{n_{l}} \exp \left(-k^{\prime} \xi_{l} \vec{r}\right)$ modulate the spherical harmonic angular functions $Y_{l m \pm}$ and $N_{1}$ is a normalization factor. The values for parameters $n=n_{1}$ and $\xi$ were chosen according to rules provided by Coppens (1997) [12].

In practice, two charge density variables, the population parameter and a kappa parameter which allows expansion and contraction of the valence shell, are added to the conventional parameters of structure analysis [13]. The population parameters $P_{v}$ and $P_{l m}$ are floated along with $\kappa^{\prime}$ and $\kappa^{\prime \prime}$ during the refinement.

The sum over $m$ in (1) includes $\pm l$, so that for each one, $2 l+1$ functions are included. The aspheric charge density was described at the octupole level $(l=3)$ for the atoms $\mathrm{C}, \mathrm{N}$, and $\mathrm{O}$ and the hexadecapole for the atoms $\mathrm{S}(l=4)$ and at the dipole levels ( $l=1$ or 2 ) for hydrogen atoms not involved and involved in strong $\mathrm{H}$ bonds, respectively. Charge densities of all hydrogen were considered to have cylindrical symmetry along the corresponding hydrogen-heavy atom bond.
TABLE 1: Refinement details.

\begin{tabular}{lccc}
\hline & $N$ & $R$ & $w R$ \\
\hline Spherical refinement & 13 & 0.0274 & 0.03997 \\
Multipole refinement & 68 & 0.02358 & 0.03165 \\
Wavelength, $\lambda(\AA)$ & 0.71073 & & \\
Completeness $(\%)$ & 99.4 & & \\
Resolution, $\operatorname{Sin} \theta / \lambda\left(\AA^{-1}\right)$ & 0.8 & & \\
\hline
\end{tabular}

$N$ is the number of refined parameters. $R=\sum\left|F_{o}\right|-\left|F_{c}\right| / \sum\left|F_{o}\right| ; w R=$ $\left[\sum w\left(\left|F_{o}\right|-\left|F_{c}\right|\right)^{2} / \sum w\left|F_{o}\right|^{2}\right]^{1 / 2}$.

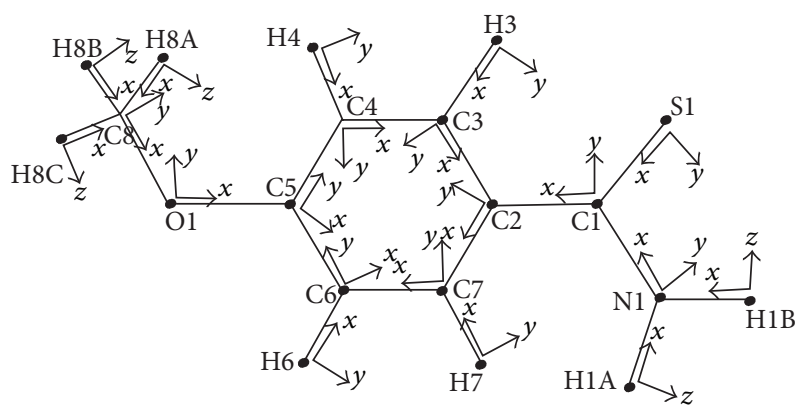

FIgURE 1: Labeling of the atoms and definition of local orthogonal reference axes for the atom-centered multipolar functions.

TABLE 2: Matrix for differences in MSDAs (mean square displacements of atoms) (values listed are $10^{4}$ MSDAs for column atom minus that for row atom; values correspond to chemical bonds).

\begin{tabular}{lcccccc}
\hline Molecule A & C5 & C1 & C8 & C2 & C7 & C4 \\
\hline S1 & & 36 & & & & \\
C3 & & & & -21 & & -19 \\
N1 & & -4 & & & & \\
C6 & 7 & & & & & \\
C4 & & & & 1 & & \\
C7 & & 16 & & & & \\
C2 & & C13 & C9 & C15 & C12 \\
\hline Molecule B & C14 & & 42 & & & \\
S2 & & & 31 & & \\
N2 & & & & & -3 \\
C11 & & & & & \\
C12 & & 7 & & & \\
C10 & & & 28 & 35 & \\
C15 & 1 & & & & \\
\hline
\end{tabular}

The quality of a refined model can be monitored based on the residuals and the goodness of fit apart from closely inspecting the deformation density maps. This model provides the advantage of using constraints based on assumed local symmetry on the atomic sites to limit the number of 
TABLE 3: Rigid-body motion parameters.

\begin{tabular}{|c|c|c|c|c|c|c|}
\hline \multicolumn{2}{|l|}{$L\left(\operatorname{rad}^{2}\right)$} & \multicolumn{2}{|r|}{$T\left(\AA^{2}\right)$} & \multicolumn{3}{|c|}{$S(\operatorname{rad} \AA)$} \\
\hline 0.00415 & $0.00036-0.00003)$ & 0.02595 & $0.00051 \quad 0.00165$ & $(-0.00001$ & -0.00055 & $-0.00002)$ \\
\hline & $0.00011-0.00009$ & & $0.05841-0.00143$ & -0.00011 & -0.00010 & 0.00050 \\
\hline ( & $0.00029 /$ & 1 & 0.03275 & -0.00016 & -0.00011 & 0.00009 \\
\hline
\end{tabular}

TABLE 4: Net charges $(q)$ in the different atoms of the title compound.

\begin{tabular}{lccc}
\hline Atoms & & $q$ & \\
& Experimental & Ab initio & Semiempirical \\
\hline S1 & -0.332 & -0.250 & -0.223 \\
O1 & -0.124 & -0.243 & -0.207 \\
N1 & -0.004 & -0.026 & -0.035 \\
C6 & 0.026 & -0.038 & -0.166 \\
C3 & -0.020 & -0.079 & -0.077 \\
C5 & 0.022 & 0.025 & -0.146 \\
C2 & 0.084 & -0.098 & 0.102 \\
C1 & -0.002 & -0.026 & -0.212 \\
C8 & 0.138 & -0.037 & -0.078 \\
C7 & -0.019 & -0.053 & 0.077 \\
C4 & 0.026 & 0.134 & -0.036 \\
H1A & 0.017 & 0.207 & 0.221 \\
H1B & 0.017 & 0.213 & 0.243 \\
H3 & 0.019 & 0.070 & 0.135 \\
H4 & 0.047 & 0.060 & 0.133 \\
H6 & 0.053 & 0.076 & 0.163 \\
H7 & 0.019 & 0.063 & 0.144 \\
H8A & 0.104 & 0.075 & 0.109 \\
H8B & 0.104 & 0.066 & 0.077 \\
H8C & 0.104 & 0.062 & 0.075 \\
\hline
\end{tabular}

least-squares variables. Figure 1 gives the local site symmetry of the different atoms of the molecule.

\section{Results and Discussion}

3.1. Structural and Thermal Vibration Analysis. The main characteristic of this structure is that the eight molecules in the unit cell are engaged in seven chains formed by infinite chains of hydrogen-bonded coplanar molecules. The different chains link translationally equivalent molecules along the $c$ crystallographic axis through $\mathrm{C}-\mathrm{S}$ and $\mathrm{N}-\mathrm{H}$ bonds.

The rigid bond test indicated that the differences between the mean square displacements amplitudes (MSDAs) along interatomic directions have magnitudes $\Delta \leq 10 \times 10^{-4} \AA^{2}$ for nearly all the bonded pairs of the non- $\mathrm{H}$ atoms [14]. In contrast, along intramolecular interatomic directions some $\Delta$ values were considerably larger, indicating that the molecule is not entirely rigid and that low-frequency soft modes of internal molecular vibration contribute significantly to the observed mean square displacements [15]. The thermal motion analysis of the title compound has been performed

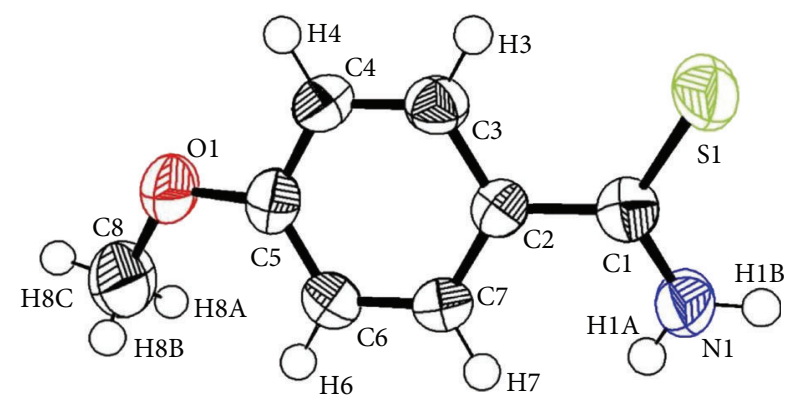

FIgURE 2: ORTEPIII diagram of the title compound. Thermal ellipsoids are shown for the $30 \%$ probability level.

using the THMA11 program [16]. Selected results are presented in Table 2.

The rigid-body motion is described by three tensors $T$, $L$, and $S$ taking into account translation, liberation, and the correlation between translation and liberation, of the rigid group, respectively. These tensors are obtained by a leastsquares fit refinement using the observed atomic thermal motion parameters obtained by the refinement.

In terms of rigid-body TLS, the calculated anisotropic thermal parameters are given in the Trueblood notation as such:

$$
U_{i j}=T_{i j}+G_{i j k l} L_{k l}+H_{i j k l} S_{k l}+D^{2} \Omega^{2} n_{i} n_{j},
$$

where $G$ and $H$ are geometrical parameters. The analysis of the non- $\mathrm{H}$ atoms in terms of rigid-body motion yields the $T$, $L$, and $S$ tensor values summarized in Table 3. The ellipsoids of the different atoms representing their thermal motion described before are shown with an ORTEPIII diagram [17] in Figure 2.

3.2. Electron Density Maps. The aspherical atom model used in multipole refinement gives structure factor phases closer to the true phases for crystals than the spherical or independent atom model does. This enables the mapping of the electron density by the Fourier synthesis in various ways using the WinGX program [18] to represent the experimental density deformation and the VMoPro program, implemented in the MoPro package, to observe the dynamic density deformation of the molecule.

The residual map using a spherical atom model is shown in Figure 3, from which we can notice the absence of the density on the atomic sites and the appearance of all the bond density peaks. This map confirms the high quality of the data 
TABLE 5: Components of the dipolar moment of the charge distribution from the point charge models (AM1 and B3LYP/6-31G*) and comparison with the components derived from experimental electron density (multipolar refinement).

\begin{tabular}{|c|c|c|c|c|c|}
\hline Methods & Models & $\mu_{x}$ & $\mu_{y}$ & $\mu_{z}$ & $\mu$ (Debye) \\
\hline X-Ray experiment & Multipolar refinement & -2.1064 & 1.5995 & 1.5281 & 3.0546 \\
\hline Semiempirical & AM1 & -1.2175 & 1.3216 & 1.3220 & 2.2308 \\
\hline $\mathrm{Ab}$ initio & B3LYP/6-31G* & -2.4646 & 2.2520 & 1.8834 & 3.8332 \\
\hline
\end{tabular}

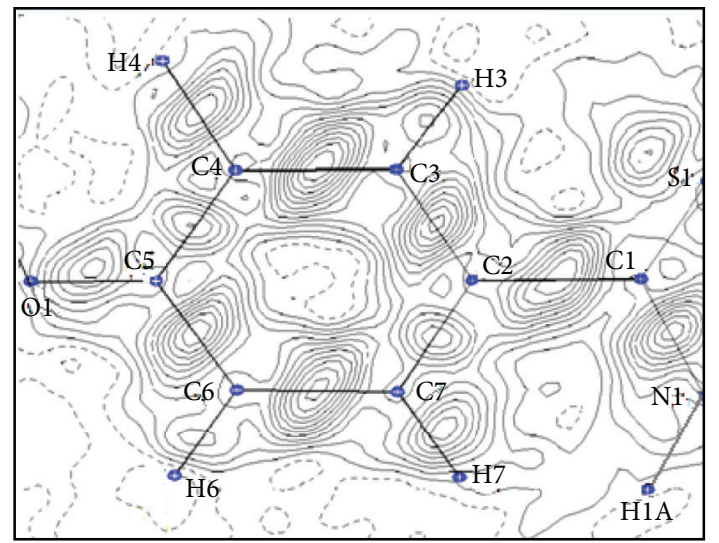

Figure 3: Experimental density map from high-order refinement. Contour map is $0.06 \mathrm{e} \cdot \AA^{-3}$.

sets and the efficiency of the formalism used for the data processing as proposed by Blessing [19].

The final electron density deformation was calculated using the following equation:

$$
\begin{aligned}
& \Delta \rho_{\text {dyn }}(\vec{r}) \\
& \quad=\frac{1}{V} \sum_{H}\left[\left|F_{\text {mul }}(\vec{H})\right| e^{i \varphi_{\mathrm{mul}}(\vec{H})}-\left|F_{\mathrm{sph}}(\vec{H})\right| e^{i \varphi_{\mathrm{sph}}(\vec{H})}\right] e^{-i 2 \pi \vec{H} \cdot \vec{r}} .
\end{aligned}
$$

This map is obtained from the calculated multipole structure factors $F_{\text {mul }}(H)$, where $F_{\text {sph }}(H)$ is computed with atomic positions and thermal parameters obtained from the multipole refinement. Figure 4 shows this electron density deformation on the different atoms in the benzene ring section of the title compound, where one can easily observe the obvious increase of the density and the good localization peaks in the middle of covalent bonds of the cycle of the molecule. In Figure 5, the density map contains two independent molecules A and B. The density maps are given in the two representations of the methoxy groups. Figure 6(a) shows the density deformation at the plane $y z$ and Figure 6(b) the deformation in the plane $x y$.

A residual density map in the molecular plane obtained in the final cycle of multipole refinement (see Figure 7) shows the adequacy of the multipolar model to describe the electron experimental density of the molecule. The quasitotality absence of the density peaks again confirms the high quality of the recorded data and the precision of the used equipment.

3.3. Hydrogen Bond. Hydrogen bonds can be classified on the basis of charge density. The crystal structure of the title compound rests on chains of molecule joined by hydrogen bonds. The almost linear hydrogen bonding links by translation of equivalent molecules along the $c$ crystallographic axis in the unit cell. In this crystal, the molecules form dimers via intermolecular $\mathrm{N}-\mathrm{H}$...S intermolecular hydrogen bonds. The dimers are further linked by $\mathrm{C}-\mathrm{H} \cdots \mathrm{O}$ hydrogen bonds into chains along the $c$-axis.

Figure 8 shows the electron density in the plane of the hydrogen bonds formed by the atoms (N2, H2B, and $\mathrm{S} 1$ ), existing in the molecule. The electron density in the intermolecular hydrogen bonds is deficient in charge between the donor and acceptor of the molecule.

3.4. Net Atomic Charges. Knowledge of the charge density function can lead to some important properties of the molecule such as the net charges on the different atoms, the molecular dipole moment, and the electrostatic potential around the molecule. The valence population coefficients $P_{v}^{i}$ were used to estimate the partial charges on the different atoms according to the following equation:

$$
q_{i}=n_{i}-P_{v}^{i}
$$

where $n_{i}$ is the total number of electrons of atoms $i$. The population coefficients are presented in Table 4 . The refined atomic charges are generally of the sign expected from chemical knowledge: $\mathrm{H}$ atoms are positively charged, whereas $\mathrm{O}, \mathrm{S}$, and $\mathrm{N}$ atoms are negatively charged. The net atomic charges obtained by the X-ray experimental method using the multipolar model are compared to those derived from the theoretical calculations. Semiempirical calculations were carried out using the AM1 (Austin's method) and ab initio calculations were carried out at the HF/6-31G ${ }^{*}$ levels of theory using the Gaussian package. The results obtained by both theory and experiment methods are summarized in Table 4. The experimental results and those derived from the semiempirical and $\mathrm{ab}$ initio calculations are in good agreement concerning the signs of the net charges of the $\mathrm{H}$ atoms and those of the $\mathrm{N}, \mathrm{S}$, and $\mathrm{O}$ atoms. 


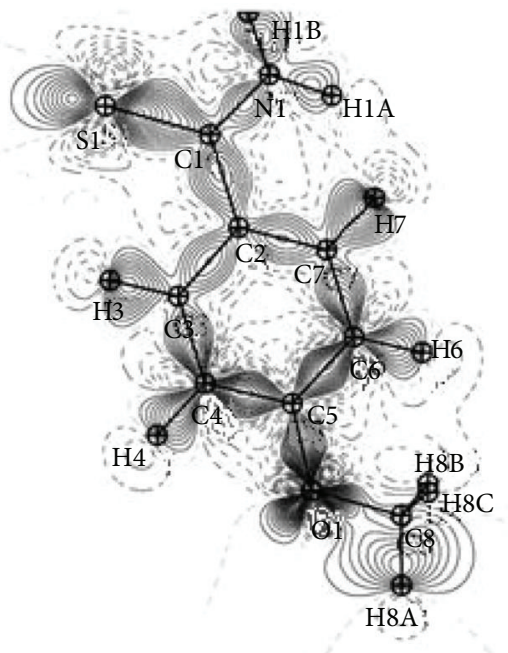

Molecule A

(a)

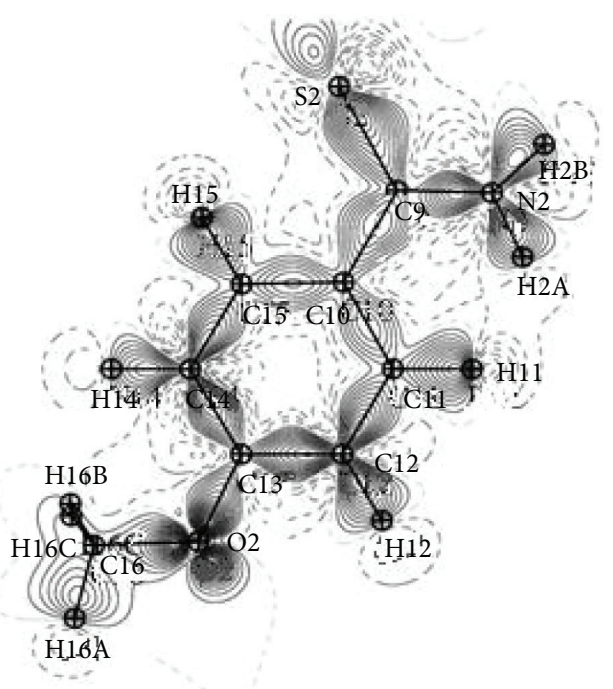

Molecule B

Figure 4: Dynamic density maps of the two molecules A and B with the methoxy groups oriented in opposite conformations of the title compound. Contour map is $0.05 \mathrm{e} \cdot \AA^{-3}$.

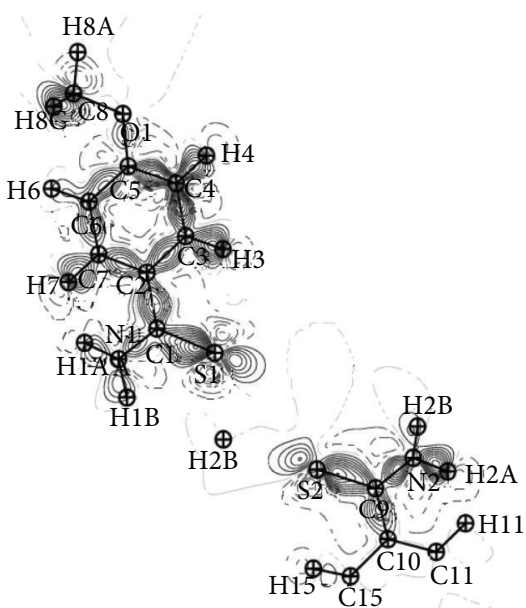

Figure 5: Dynamic density map in the plane ( $\mathrm{S} 1, \mathrm{C} 1$, and N1) contains two independent molecules $\mathrm{A}$ and $\mathrm{B}$. Contour map is $0.05 \mathrm{e} \cdot \AA^{-3}$.

3.5. Molecular Dipole Moment. The molecular dipole moment was first estimated using the population coefficients of the multipolar density model from the following equation:

$$
\mu=\sum_{i} q_{i} r_{i}+\sum_{i} \frac{4 n+3}{3 k_{i}^{\prime \prime}}\left(P_{x i} i+P_{y i} j+P_{z i} k\right),
$$

where $r_{i}$ is the atomic position and $n$ is the number of electrons in the molecule. $P_{i}$ are the dipolar electron populations. Dipole moment orientation in the molecule is shown in Figure 9. The resulting molecular dipole moment direction is in accordance with the evaluation of the positive sign of the net charges on the $\mathrm{H}$ atoms and the negative sign of the net charges on the $\mathrm{O}, \mathrm{S}$, and $\mathrm{N}$ atoms.

In a parallel study, the net charges on the different atoms and the molecular dipole moment were also estimated by ab initio (B3LYP/6-31G ${ }^{*}$ ) and semiempirical (AM1) calculations. In this procedure the treatment is confined to the valence-shell electrons. The results of these calculations are presented in Table 5.

From the results, we can see a good agreement between the value obtained with X-ray diffraction (3.0546 D) and the other values obtained by theoretical calculation (AM1 gives 2.2308 D and DFT gives 3.8332 D).

Analysis of obtained dipole moments shows that the size and direction of the dipole moment vector depend mainly on the position of substituents. Furthermore, the orientation of the dipole moment vector is toward the sulfur atom.

3.6. Electrostatic Potential. The distribution of positive and negative charges in a crystal fully defines physical properties like the electrostatic potential and its derivatives. The electrostatic potential is important in the study of intermolecular interactions of molecular systems.

For a continuous charge distribution study, the potential is obtained by integration over the space containing the distribution. At a point defined by $r^{\prime}$, the potential in given by

$$
\phi\left(r^{\prime}\right)=\int \frac{\rho_{\operatorname{total}(r)}}{\left|r-r^{\prime}\right|} d r .
$$

In which $\rho_{\text {total }}$ represents both the nuclear and the electronic charge, where the integration is over the molecular volume, 


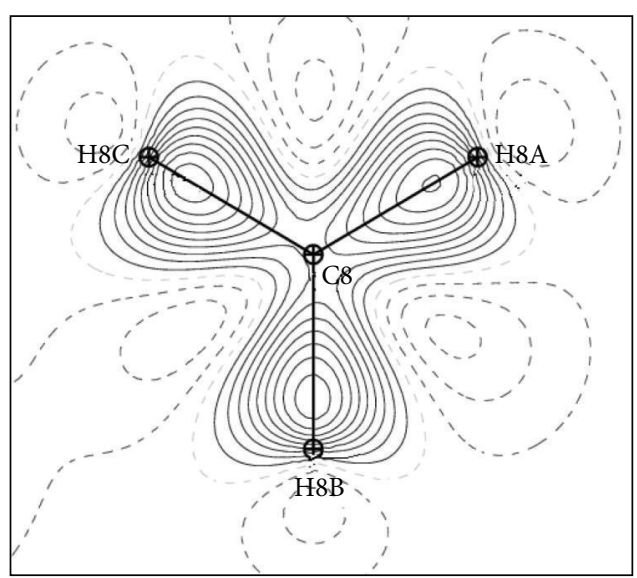

(a)

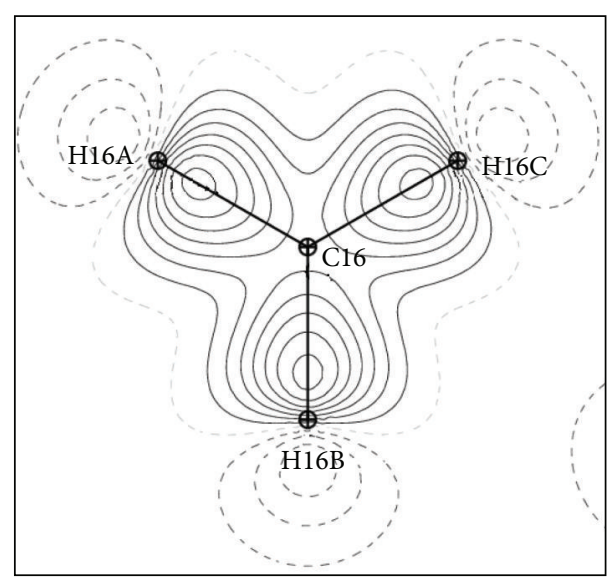

(b)

FIGURE 6: Dynamic density map of the methoxy groups: (a) deformation in the plane $x y$ of the molecule A; (b) deformation in the plane $x y$ of the molecule B. Contour map is $0.05 \mathrm{e} \cdot \AA^{-3}$.

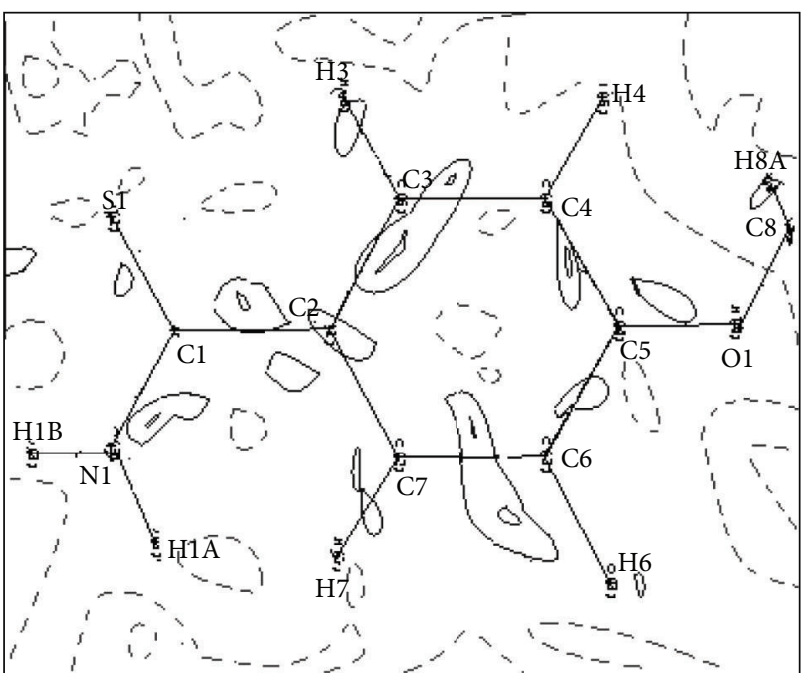

Figure 7: Residual density map: $\rho_{\text {res }}=\rho_{0}-\rho_{\text {mult }}$. Contour map is $0.05 \mathrm{e} \cdot \AA^{-3}$

and $r$ represents the atomic position relative to common origin.

The integration includes the atoms of only one molecule and therefore does not include directly the effects of charge distribution of the neighboring molecules.

Figure 10 shows the electrostatic potential distribution in the plane of the base ring. The extension of the positive electrostatic potential around the carbothioamide and C$\mathrm{H}$ groups and the regions of negative electrostatic potential around the nitro and hydroxyl groups confirm the nature of the intramolecular charge transfer as found by the orientation of the molecular dipole moment.

The obtained point charges corresponding to $S$ atom are $-0.332,-0.250$, and -0.223 e from experimental, ab initio, and semiempirical methods, respectively. But the region very close to $\mathrm{S}$ appears positive. This is because of the fact that $\mathrm{S}$ atom of the title compound is surrounded by the most

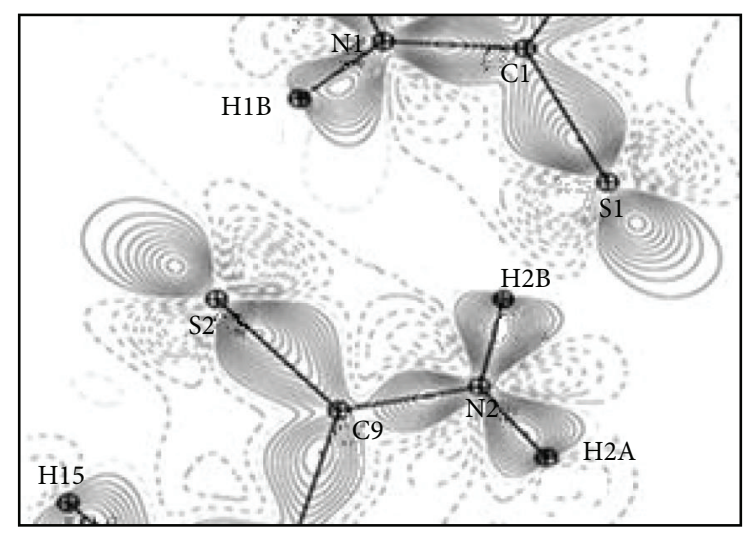

FIGURE 8: Deformation density map in the plane of the hydrogen bond formed by the atoms $(\mathrm{N} 2, \mathrm{H} 2 \mathrm{~B}$, and $\mathrm{S} 1)$. Contour map is $0.05 \mathrm{e} \cdot \AA^{-3}$.

electropositive hydrogen atoms $(\mathrm{H} 1 \mathrm{~A}=0.017, \mathrm{H} 1 \mathrm{~B}=0.017, \mathrm{H} 3$ $=0.019$, and $\mathrm{H} 7=0.019 \mathrm{e}$ ).

\section{Conclusion}

This study has obtained good accurate results on the structure which gives high-quality descriptive model for the electron charge density distribution from X-ray diffraction experiment. It also shows that electron density can yield electronic proprieties such as dipole moment. Its determination lets us locate the region of the donor and the acceptor groups and also the direction of charge transfer within the molecule. The deformation of the electrostatic potential of the title compound shows that the electronegative potential is located on the side of methoxy group and electropositive potential is on the side of carbothioamide group.

In the multipole refinement, all nonhydrogen atoms were refined anisotropically and subject to a rigid-bond constraint. $\mathrm{H}$ atoms anisotropic parameters are obtained from TLS 


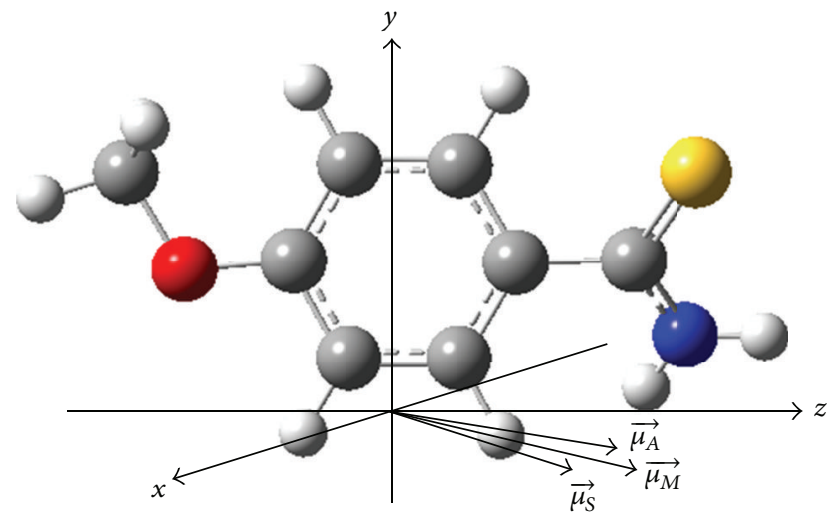

FIGURE 9: Orientation of the molecular dipole moment of the title compound; the origin is at the center of mass of the molecule; $\mu_{M}$ : molecular dipole moment from the multipolar model; $\mu_{S}$ : molecular dipole moment from the semiempirical calculation; $\mu_{A}$ : molecular dipole moment from the ab initio calculation.

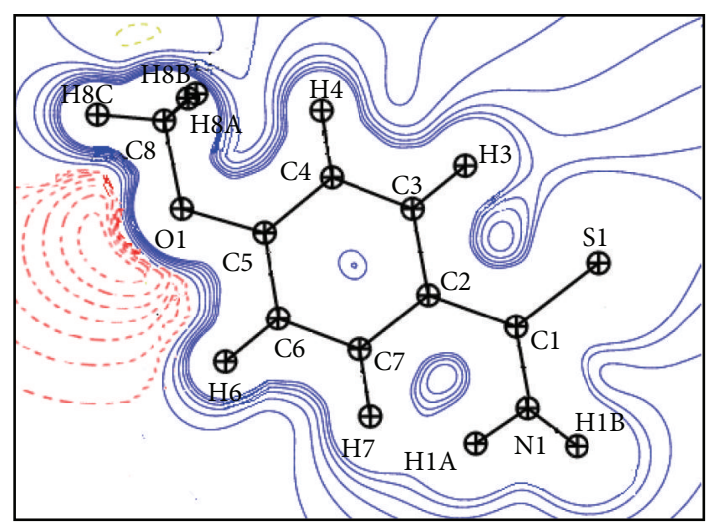

FIgURE 10: The electrostatic potential maps around the molecule. The section is in the plane of the benzene ring.

analysis. The final $R$ factor (2.2\%) attests to the quality of the data.

To study the effect of electron charge transfer in biological activity more, we should perform vibrational analysis using FT-Raman and FTIR spectroscopic techniques.

\section{References}

[1] H. Quiniou and O. Guillton, "1,3-Thiazines," in Advances in Heterocyclic Chemistry, A. R. Katritzky, Ed., vol. 50, pp. 85-156, Elsevier, San Diego, Calif, USA, 1990.

[2] S. P. Singh, S. S. Parmar, K. Raman, and V. I. Stenberg, "Chemistry and biological activity of thiazolidinones," Chemical Reviews, vol. 81, no. 2, pp. 175-203, 1981.

[3] M. D. Mashkovskii, "Drugs," Torsing, vol. 2, p. 244, 1998 (Russian).

[4] S. T. Lebana, R. Sultana, and G. Hendal, "Heterocyclic thioamides of copper(I): synthesis and crystal structures of copper complexes with 1,3-imidazoline-2-thiones in the presence of triphenyl phosphine," Polyhedron, vol. 27, no. 3, pp. 1008-1016, 2008.
[5] S. Ali, S. Hameed, A. Luqman, T. Akhtar, and M. Parvez, "4Methoxybenzenecarbothioamide," Acta Crystallographica Section E, vol. 66, no. 6, p. o1272, 2010.

[6] G. M. Sheldrick, SHELXL97: Program for Crystal Structure Determination, University of Gttingen, Göttingen, Germany, 1997.

[7] N. K. Hansen and P. Coppens, "Testing aspherical atom refinements on small-molecule data sets," Acta Crystallographica A, vol. 34, pp. 909-921, 1978.

[8] C. Jelsch, B. Guillot, A. Lagoutte, and C. Lecomte, "Advances in protein and small-molecule charge-density refinement methods using MoPro," Journal of Applied Crystallography, vol. 38, no. 1, pp. 38-54, 2005.

[9] M. J. Frisch, G. W. Trucks, H. B. Schlegel et al., GAUSSIAN-03, Revision E.01, Gaussian, Wallingford, UK, 2004.

[10] E. Prince and A. J. C. Wilson, International Tables for X-Ray Crystallography, vol. C, Kluwer Academic, Boston, Mass, USA, 2nd edition, 1999.

[11] R. F. Stewart, E. R. Davidson, and W. T. Simpson, "Coherent Xray scattering for the hydrogen atom in the hydrogen molecule," The Journal of Chemical Physics, vol. 42, no. 9, pp. 3175-3187, 1965.

[12] P. Coppens, X-Ray Charge Densities and Chemical Bonding, Oxford, New York, NY, USA, 1997.

[13] M. A. Spackman, "5 Charge densities from X-ray diffraction data," Annual Reports on the Progress of Chemistry C, vol. 94, pp. 177-207, 1998.

[14] F. L. Hirshfeld, "Can X-ray data distinguish bonding effects from vibrational smearing?" Acta Crystallographica A, vol. 32, pp. 239-244, 1976.

[15] R. E. Rosenfield, K. N. Trueblood, and J. D. Dunitz, "A test for rigid-body vibrations based on a generalization of Hirshfeld's "rigid-bond" postulate," Acta Crystallographica A, vol. 34, pp. 828-829, 1978.

[16] K. N. Trueblood, THMA11 Program, Department of chemistry and biochemistry, University of California, Los Angeles, Calif, USA, 1990.

[17] L. J. Farrugia, "ORTEP-3 for windows-a version of ORTEPIII with a graphical user interface (GUI)," Journal of Applied Crystallography, vol. 30, no. 5, p. 565, 1997.

[18] L. J. Farrugia, "WinGX suite for small-molecule single-crystal crystallography," Journal of Applied Crystallography, vol. 32, no. 4, pp. 837-838, 1999.

[19] R. H. Blessing, "Outlier treatment in data merging," Journal of Applied Crystallography, vol. 30, no. 4, pp. 421-426, 1997. 

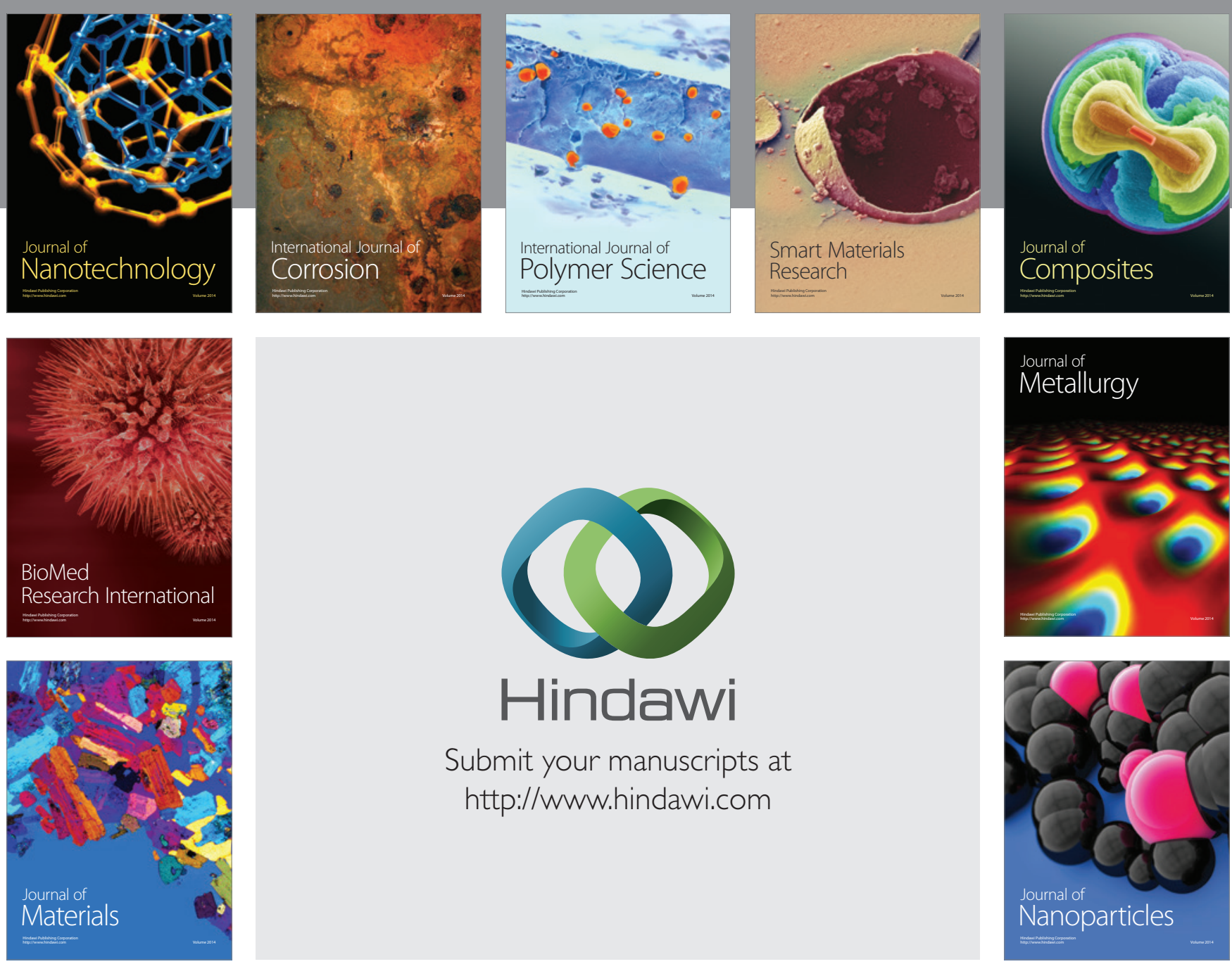

Submit your manuscripts at http://www.hindawi.com
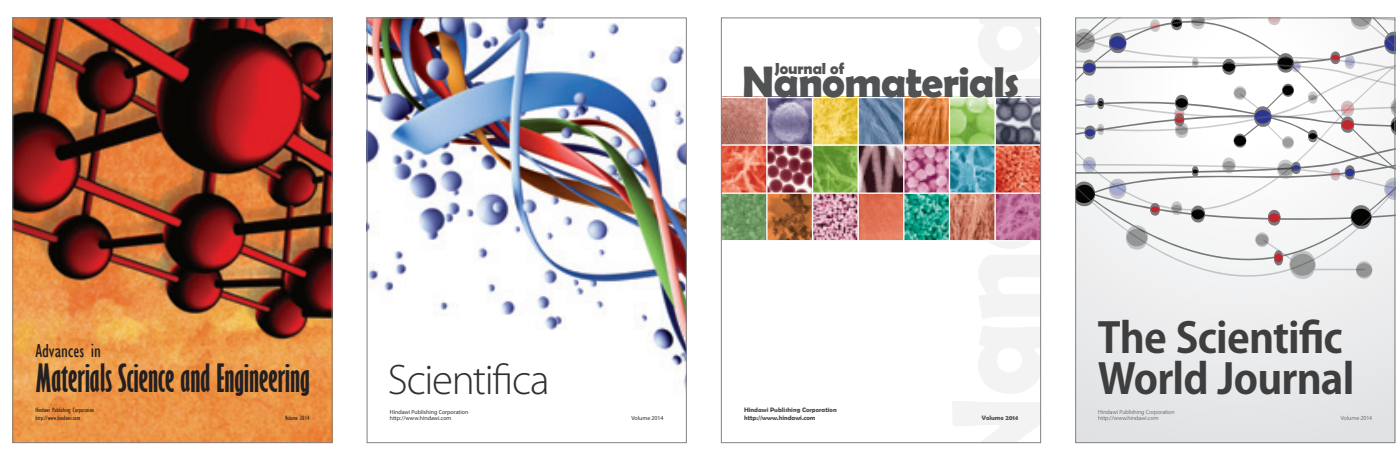

\section{The Scientific World Journal}
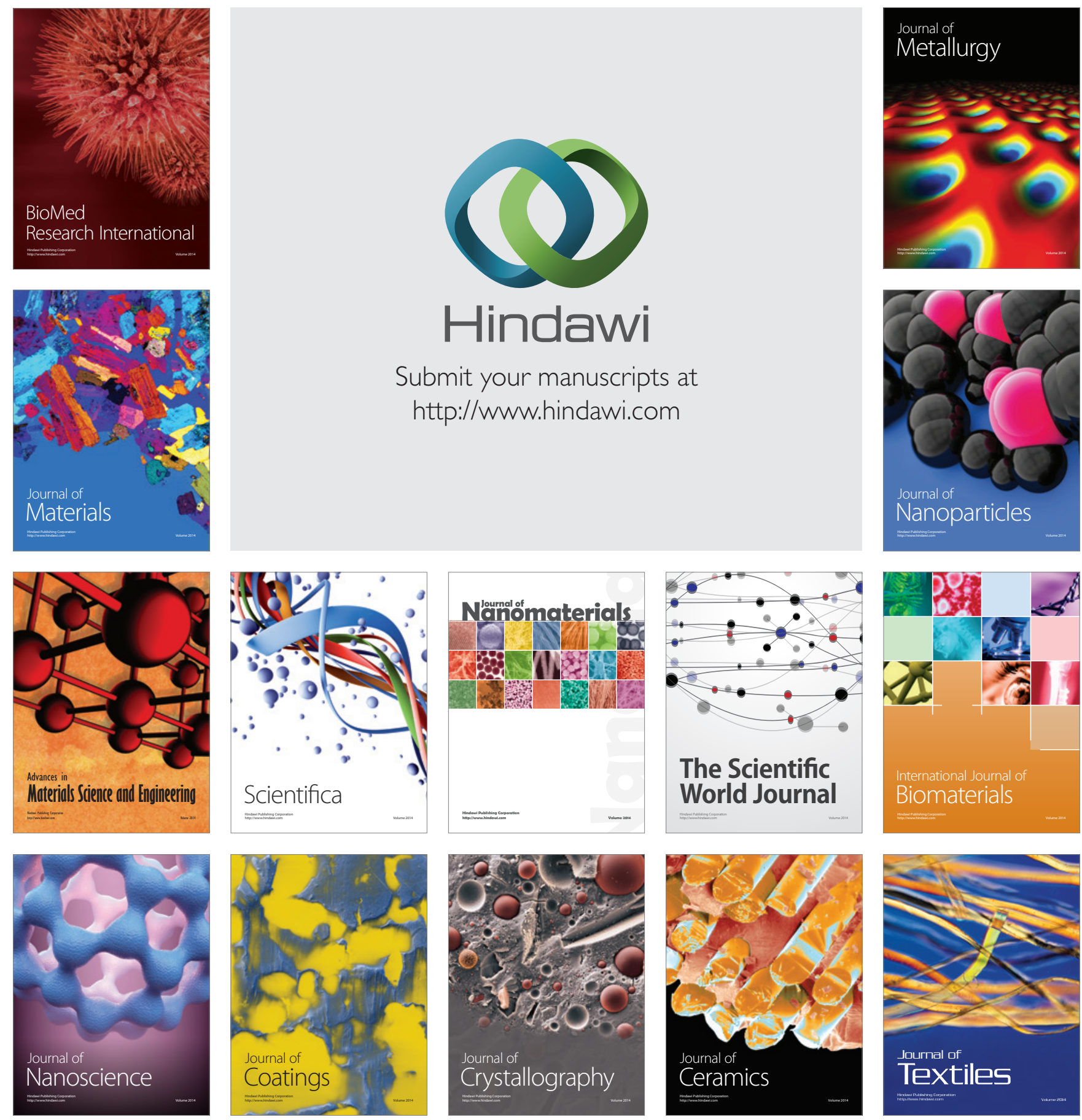